Research Paper

\title{
Overexpression of $\alpha$-sma-positive fibroblasts (CAFs) in Nasopharyngeal Carcinoma Predicts Poor Prognosis
}

\author{
Jiewei Chen ${ }^{1,2^{*}}$, Pengfei Yang ${ }^{3 *}$, Yongbo Xiao ${ }^{1 *}$, Yijun Zhang ${ }^{1}$, Jun Liu ${ }^{1}$, Dan Xie ${ }^{1,2}$, Muyan Cai ${ }^{1,2}$, Xinke \\ Zhang ${ }^{1,2 \bowtie}$ \\ 1. Department of pathology, Sun Yat-Sen University Cancer Center, 510060 Guangzhou, China; \\ 2. State Key Laboratory of Oncology in South China, Collaborative Innovation Center for Cancer Medicine; \\ 3. Department of Pathology, The Fifth Affiliated Hospital, Sun Yat-Sen University, Zhuhai, 519000, China; \\ *Contributed equally \\ $\square$ Corresponding author: Xinke Zhang, Department of Pathology, Sun Yat-sen University Cancer Center, No. 651, Dongfeng Road East, 510060 Guangzhou, \\ China. Tel.: 86-20-87342270; zhangxk@sysucc.org.cn \\ (c) Ivyspring International Publisher. This is an open access article distributed under the terms of the Creative Commons Attribution (CC BY-NC) license \\ (https:// creativecommons.org/licenses/by-nc/4.0/). See http://ivyspring.com/terms for full terms and conditions.
}

Received: 2017.03.29; Accepted: 2017.08.03; Published: 2017.10.23

\begin{abstract}
Purpose: The aim of this study is to investigate the differential expression of $\alpha$-sma-positive fibroblasts (CAFs) in nasopharyngeal carcinomas (NPCs), nasopharyngitis, metastatic tissues of NPCs and its prognostic value in NPCs.

Methods: The expression of $\alpha$-sma-labeled CAFs in 85 NPCs, 32 nasopharyngitis and 12 metastatic tissues of NPCs was detected by immunohistochemical method. The relationship between CAFs and clinicopathological parameters of NPCs was analyzed.

Results: The high density of CAFs in the NPCs, nasopharyngitis and metastatic tissues of NPCs group were $41.2 \%(35 / 85), 6.2 \%(2 / 32)$ and $83.3 \%$ (10/12), and a significant difference was showed among these three groups $(P<0.05)$. Chi-square test showed that there was no significant correlation between the density of CAFs and gender, age, $N$ stage, treatment $(P>0.05)$, but closely correlated with $T$ stage and relapse $(P<0.05)$. Kaplan-Meier survival analysis showed that the mean overall survival of high-density and low-density CAFs was 86.8 months and 127.0 months, respectively. Correspondingly, the 5-year survival rates were $57.1 \%(20 / 35)$ and $90.0 \%(45 / 50)$, and there were inversely statistical differences between two groups $(P<0.05)$. Cox multivariate analysis showed that the density of CAFs could be used as an independent prognostic factor for the survival of NPC patients $(P<0.05)$.

Conclusions: The density of CAFs could be closely related to the metastasis of NPCs, and also is an efficient prediction factor of poor survival in patients with NPCs.
\end{abstract}

Key words: a-sma, Nasopharyngeal Carcinomas, CAFs, Prognosis

\section{Introduction}

Nasopharyngeal carcinoma (NPC) is a kind of common malignancy in South China and Southeast Asia [1]. Its etiology is related to Epstein-Barr (EB) virus infection, genetic susceptibility, interaction between various oncogenes and tumor suppressor genes, environment and living customs and so on [2]. Because of the low outpatient rate at its early stage, the majority of NPC patients of in-hospital have reached III and IV stage, with a 5-year survival rate of $50 \%$ to $60 \%$ [3]. Carcinoma-associated fibroblasts (CAFs) have been generally considered to be spindle fibroblast-like stromal cells with expression of a-smooth muscle actin (a-sma) [4]. As a major and important component of tumor matrices, CAFs play an important role in tumor development, and promote tumorigenesis, invasiveness and metastasis by stimulating angiogenesis [5]. Several studies have shown that CAFs can be used as important prognostic factors in a variety of tumors [6-8]. However, its clinical significance in the prognostic value of NPCs has rarely reported. In this study, the expression of a-sma protein in NPC tissues was detected by 
immunohistochemistry and the association of its expression with clinicopathological characteristics was analyzed in NPCs by statistical analysis.

\section{Materials and Methods}

\section{Ethics statement}

The study was approved by the Institute Research Medical Ethics Committee of Sun Yat-sen University. No informed consent (written or verbal) was obtained for use of retrospective tissue samples from the patients within this study, most of whom were deceased, since this was not deemed necessary by the Ethics Committee, who waived the need for consent. All samples were anonymised.

\section{Patients and tissue specimens}

We collected paraffin samples from September 2002 to November 2004 in Department of Pathology, Sun Yat Sun University Cancer Center, the number of the pathological diagnosis of NPC, nasopharyngitis and Metastatic tissues of NPC were 85 cases, 32 cases, 12 cases, respectively. The NPC group contained 65 males $(76.5 \%)$ and 20 females $(23.5 \%)$, with a median age of 44 and a mean follow-up time of 90.8 months (the median follow-up time was 110 months). The diagnostic criterion of all cases in this study was based on the 2000 WHO criteria for tumor classification, and tumor staging was on the basis of UICC (International Union against Cancer) and the AJCC (American Joint Committee on Cancer).

\section{Immunohistochemistry (IHC)}

The immunohistochemical staining of a-Smooth muscle actin (a-sma) protein was done according to standard EnVision ${ }^{\mathrm{TM}}$ procedure. We sliced the paraffin blocks into 3- $\mu \mathrm{m}$ sections and dry sections in incubator at $60^{\circ} \mathrm{C}$. After deparaffinized with xylene and rehydrated in a graded ethanol series, the slides were placed in boiled citric acid buffer for antigen retrieval. We incubated the sections with first antibody a-sma (Sigma, A5228, operative solution concentration $1: 12000$ ) at $37^{\circ} \mathrm{C}$ for $50 \mathrm{~min}$. After immersed in $3 \%$ hydrogen peroxide for $10 \mathrm{~min}$ to inhibit the endogenous peroxidase activity, the sections were incubated with secondary antibody (DAKO, K5007) at $37^{\circ} \mathrm{C}$ for $30 \mathrm{~min}$. Then, the color reaction was developed by 3,3-diaminobenzidine $(\mathrm{DAB})$ and the degree of color development was controlled under microscope. Finally, the sections were counterstained with hematoxylin, dehydrated in a graded ethanol series, cleared in xylene, and mounted with neutral gum. Positive and negative controls were set in the staining procedure.

\section{IHC evaluation}

Positive or negative fibroblasts were identified on the basis of a-sma expression. The positive expression of a-sma protein is brownish yellow or chocolate brown in cytoplasm. Selecting 4 typical visual field of tumor stroma in every slice by high-power field of the microscope, we counted the area of a-sma-positive fibroblasts (CAFs) and calculated CAFs density (dCAFs) =the positive staining area/tumor stromal area (\%) of every visual field (vascular positive staining as internal control was not counted) [8]. CAFs density is the mean value of the 4 fields. With double-blind method, staining score analyses were done by two independent and experienced pathology doctors. A more experienced pathology doctor would reevaluate the conflicting results.

\section{Statistical Analysis}

Statistical analysis was performed using SPSS20.0 and the cut-off value of expression level was defined as the median of clinical commonly usage [9].The difference of CAFs density between the NPC tissues, the nasopharyngitis tissues and the metastatic tissues of NPCs, the relevance between the density of CAFs and the clinicopathological parameters of NPC patients were both verified by Chi-square test. The survival analysis of NPC patients was evaluated by the Kaplan-Meier method with log-rank test. Multivariate analyses were performed using Cox proportional hazard model. All $P$ values were reported by two-sided analyses and $P<0.05$ stands for the statistical significance level.

\section{Result}

Differential expression of CAFs in NPC tissues, nasopharyngitis tissues and metastatic tissues of NPCs by immunohistochemistry

There are different expression situation for CAFs in these three kinds of tissue (Figure 1). A median of $10 \%$ were served as the cut-off value of the CAFs expression level. CAFs are analyzed in NPC tissues, nasopharyngitis tissues and metastatic tissues of NPCs, and the high density rate is $41.2 \%$ (35/85), 6.2\% $(2 / 32)$ and $83.3 \%(10 / 12)$ respectively. There are significant differences among them by Chi-square Test $(P<0.05)$ (Table 1$)$.

\section{The relation between the expression level of CAFs in NPC tissues and clinical pathological parameters}

Chi-square Test shows that the density of CAFs is significantly correlated with relapse $(P=0.023)$, and T stage $(P=0.005)$. But no significant correlation with 
other clinical pathological parameters (Gender, age, lymph node metastasis, treatment modality, $P>0.05$ ) was found. (Table 2)

Table 1. The expression of $\alpha$-sma in the NPCs, Nasopharyngitis and Metastatic tissues of NPCs

\begin{tabular}{|c|c|c|c|c|}
\hline \multirow[t]{2}{*}{ Variable } & \multicolumn{4}{|l|}{ CAFs } \\
\hline & All cases & Low density & High density & $P$ value a \\
\hline Tissue type & & & & 0.000 \\
\hline NPCs & 85 & $50(58.8 \%)$ & $35(41.2 \%)$ & \\
\hline Nasopharyngitis & 32 & $30(93.8 \%)$ & $2(6.2 \%)$ & \\
\hline $\begin{array}{l}\text { Metastatic tissues of } \\
\text { NPCs }\end{array}$ & 12 & $2(16.7 \%)$ & $10(83.3 \%)$ & \\
\hline
\end{tabular}

Table 2. Correlation of CAFs with patients' clinicopathological features in nasopharyngeal carcinomas

\begin{tabular}{|c|c|c|c|c|}
\hline \multirow[t]{2}{*}{ Variable } & \multicolumn{4}{|c|}{ CAFs } \\
\hline & All cases & Low density & High density & $P$ value a \\
\hline Age (years) b & & & & 0.054 \\
\hline$\leq 45.4$ & 47 & $32(68.1 \%)$ & $15(31.9 \%)$ & \\
\hline$>45.4$ & 38 & $18(47.4 \%)$ & $20(52.6 \%)$ & \\
\hline Gender & & & & 0.521 \\
\hline Male & 65 & $37(56.9 \%)$ & $28(43.1 \%)$ & \\
\hline Female & 20 & $13(65.0 \%)$ & $7(35.0 \%)$ & \\
\hline T stage & & & & 0.005 \\
\hline T1 And T2 & 32 & $25(78.1 \%)$ & $7(21.9 \%)$ & \\
\hline T3 And T4 & 53 & $25(47.2 \%)$ & $28(52.8 \%)$ & \\
\hline Lymph node & & & & 0.270 \\
\hline No & 17 & $12(70.6 \%)$ & $5(29.4 \%)$ & \\
\hline N1,N2 And N3 & 68 & $38(55.9 \%)$ & $30(44.1 \%)$ & \\
\hline Therapy ${ }^{c}$ & & & & 0.793 \\
\hline Therapy1 And Therapy2 & 62 & $37(59.7 \%)$ & $25(40.3 \%)$ & \\
\hline Therapy3 And Therapy 4 & 23 & $13(56.5 \%)$ & $10(43.5 \%)$ & \\
\hline Relapse & & & & 0.023 \\
\hline Yes & 11 & $3(27.3 \%)$ & $8(72.7 \%)$ & \\
\hline No & 74 & $47(63.5 \%)$ & $27(36.5 \%)$ & \\
\hline
\end{tabular}

\section{The relationship between the expression of CAFs and prognosis of NPC patients}

The univariate analysis demonstrated that these factors including age $(P=0.027), \mathrm{T}$ stage $(P=0.003)$, relapse $(P=0.000)$ and density of CAFs $(P=0.000)$ were inversely associated with the prognosis of NPC patients (Table 3). We introduce above significant factors into COX regression model to carry out multivariate analysis. The result shows that age is not independent prognostic risk factors $(P>0.05)$, while the density of CAFs, $\mathrm{T}$ stage and relapse can be identified as independent prognostic factors $(P<0.05)$ for the prognosis of the NPC patients (Table 4). Kaplan-Meier analysis demonstrated that the mean overall survival of patients with low CAFs density and patients with high CAFs density are 127.0 months and 86.8 months $(P=0.000$, Figure $2 \mathrm{~A})$. And revealed that the mean progression-free survival are respectively 130.1 months and 97.2 months $(P=0.006$,
Figure 2B). Life table analysis showed that the 5 year survival of patients with low CAFs density and high CAFs density are respectively $90.0 \%(45 / 50)$ and $57.1 \%(20 / 35)(P=0.000)$.

\section{Discussion}

a-sma, the subtype of a-actin genetically coded by ACTA2, is a member of the highly conserved actin family. It plays a key role in cell movement, structure and integrity. a-sma, reflecting the expression of CAFs in mesenchyme, is the most common marker of CAFs and does not express on normal nasopharyngitis epithelial cells or mesenchymal fibroblasts [10]. CAFs have been identified to be the most abundant cells in the tumor microenvironment [11]. CAFs is one of the major components of the epithelial-mesenchymal transition (EMT) [8] and may be associated with EMT of tumor cells and lymphogenesis of metastatic microenvironment [12]. EMT, which promotes the mesenchymal fibrosis and the tumor progressiveness [13], is a critical factor during invasion and metastases of cancer cells [14]. Raghu Kalluri et al, [15] showed that matrix microenvironment was vital to the tumorigenesis, development and metastases.

Table 3. Univariate analysis of clinicopathologic variables in 85 patients with nasopharyngeal carcinomas (log-rank test)

\begin{tabular}{|c|c|c|c|c|}
\hline Variable & All cases & $\begin{array}{l}\text { Mean } \\
\text { survival(months) }\end{array}$ & $\begin{array}{l}\text { Median } \\
\text { survival(months) }\end{array}$ & $\begin{array}{l}P \\
\text { value }\end{array}$ \\
\hline Age (years) a & & & & 0.027 \\
\hline$\leq 45.4$ & 47 & 122.0 & $\mathrm{~N}$ & \\
\hline$>45.4$ & 38 & 101.4 & & \\
\hline Gender & & & & 0.371 \\
\hline Male & 65 & 112.1 & $\mathrm{~N}$ & \\
\hline Female & 20 & 118.0 & & \\
\hline T stage & & & & 0.003 \\
\hline $\mathrm{T} 1$ And $\mathrm{T} 2$ & 32 & 128.6 & $\mathrm{~N}$ & \\
\hline T3 And T4 & 53 & 103.3 & 121.0 & \\
\hline Lymph node & & & & 0.217 \\
\hline No & 17 & 125.5 & $\mathrm{~N}$ & \\
\hline N1,N2 And N3 & 68 & 110.5 & & \\
\hline Therapy $\mathrm{b}$ & & & & 0.475 \\
\hline $\begin{array}{l}\text { Therapy } 1 \text { And } \\
\text { Therapy } 2\end{array}$ & 62 & 111.4 & $\mathrm{~N}$ & \\
\hline $\begin{array}{l}\text { Therapy3 And } \\
\text { Therapy } 4\end{array}$ & 23 & 119.4 & & \\
\hline Relapse & & & & 0.000 \\
\hline Yes & 11 & 78.7 & 67.0 & \\
\hline No & 74 & 119.0 & $\mathrm{~N}$ & \\
\hline CAFs & & & & 0.000 \\
\hline Low density & 50 & 127.0 & $\mathrm{~N}$ & \\
\hline High density & 35 & 86.8 & 108.0 & \\
\hline
\end{tabular}

This study was initially to use the density of CAFs as an evaluation index for patient's prognosis in NPCs. Our results found the density of CAFs in NPC tissues was significantly higher than that in 
nasopharyngitis tissues. In addition, the density of CAFs in metastatic tissues of NPCs was also significantly higher than that of local region. Patients with high density of CAFs had worse overall survival than that with low density. The CAFs in mesenchyme of NPC tissues in this study has preliminarily revealed that its importance in tumorigenesis, tumor metastases and predicting the prognosis of NPC patients.

Table 4. COX multivariate analysis of prognostic factors on overall survival

\begin{tabular}{llll}
\hline Variable & $\begin{array}{l}\text { Hazards } \\
\text { ratio }\end{array}$ & $95 \%$ CI & $P$ value \\
\hline Age (years) a (<=45.4 vs. $>45.4)$ & 2.083 & $0.861-5.043$ & 0.104 \\
T stage (T1-T2 vs. T3-T4) & 4.656 & $1.276-16.991$ & $\mathbf{0 . 0 2 0}$ \\
Relapse (No vs. Yes) & 4.760 & $1.698-13.342$ & $\mathbf{0 . 0 0 3}$ \\
CAFs density (Low vs. High) & 3.108 & $1.104-8.753$ & $\mathbf{0 . 0 3 2}$ \\
\hline
\end{tabular}

a Mean age.

This research detected the density of CAFs in NPC tissues, nasopharyngitis tissues and metastatic tissues of NPCs by immunohistochemistry. The results showed that the rates of high density of CAFs in the three groups are $41.2 \%, 6.2 \%$ and $83.3 \%$ respectively, and a significantly statistical difference was displayed among them, indicating that CAFs have an important role in development and metastases of NPCs, which is consistent with the results of previous researches. CAFs is the major and important component of tumor stroma which plays an important role in the growth and development of epithelial tumor $[16,17]$ by stimulating angiogenesis, secreting soluble factors (eg, growth factors, inflammatory cytokines, and reconstructing extracellular matrix (ECM) and tumor metabolites, adjust the motility and pluripotency $[18,19]$ of the tumor to make early-stage preparations for metastases.

We further revealed that the density of CAFs had a significant correlation with T stage in NPC tissues, and the mean overall survival for patients with low CAFs density is inversely longer than that of patients with high CAFs density. This result is in accordance with $\mathrm{Yu}$ feng Cheng's team's findings in their esophageal squamous cell carcinoma research which showed that the 5-year survival rate of groups with higher expression of a-SMA was significantly lower [20]. In addition, Susumu Saigusa's research on rectal cancer [21] and Ibrahim O. Bello's research on mobile tongue cancer [22] both proved that CAFs correlate with poor prognosis, and also are related to the tumor malignant degree, tumor growth and progressiveness. Similarly, some studies have showed that stromal CAFs are associated with a high risk of recurrence and worse prognosis in other solid cancers including colorectal and breast cancer [23, 24] Multivariate analysis in our research indicated that CAFs density was served as independent prognostic factor for NPCs. The similar conclusion was also reported for other epithelial tumors [20]. The evidences above revealed CAFs' important role in the occurrence, development, metastasis and prognostic evaluation of NPCs. Moreover, targeting stromal CAFs inhibited the growth of cholangiocarcinoma and improved survival of host in an experimental study [25]. Therefore, an approach that targets CAFs could be a novel strategy for cancer therapies in the future. However, the molecular mechanism of CAFs in the progressiveness and metastases of NPCs remained unclear. Thus further researches on mechanism of molecular pathway would be a meaningful attempt.

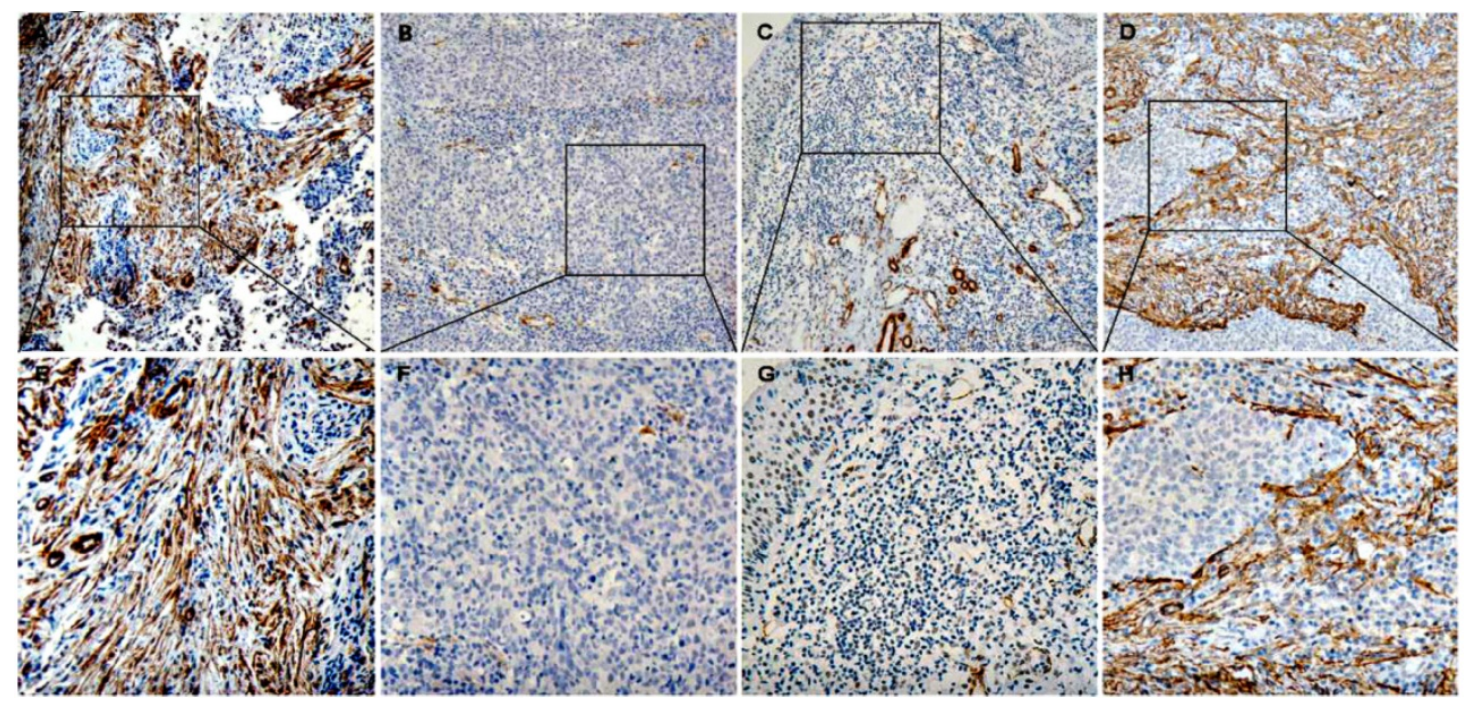

Figure 1. The expression of $\alpha$-sma in nasopharyngeal tissues $(A, E)$ is the expression of $\alpha$-sma in nasopharyngeal carcinoma tissues (CAFs high density), ( $B$, $F$ ) ( $C A F s$ low density); $(C, G)$ is the expression in nasopharyngeal tissues (CAFs low density); $(D, H)$ is the expression in the metastatic tissues of nasopharyngeal carcinoma (CAFs high density) 
A

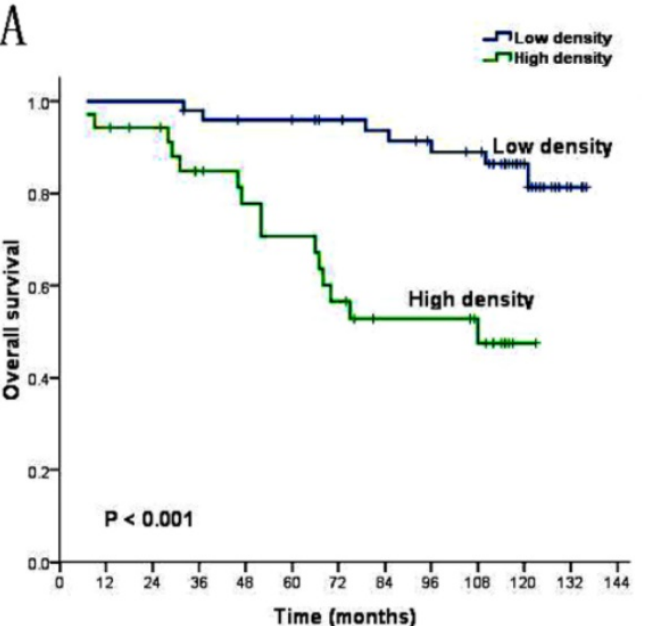

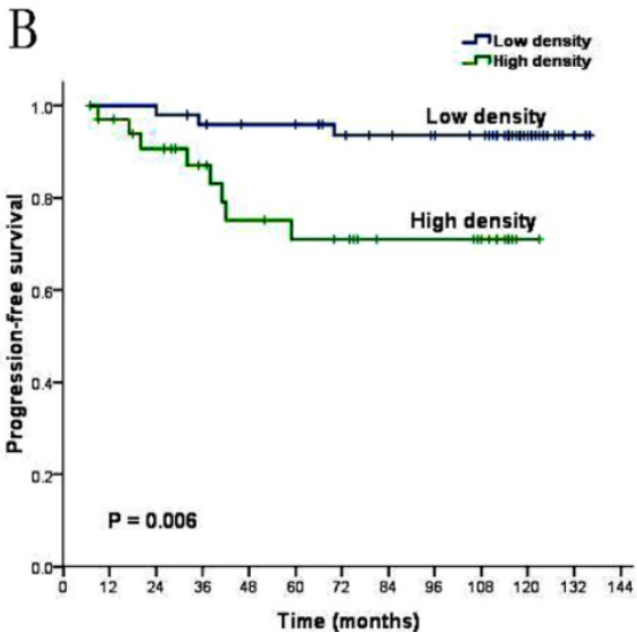

Figure 2. CAFs Kaplan-Meier survival analysis curves (A) overall survival (B) progression-free survival.

In this study, we also found a rather curious phenomenon, that is, a small amount of NPC cells really expressed a-sma. This indicated some of the NPC cells could have achieved the characteristics of CAFs, suggesting a stronger metastatic ability has existed in these NPC cells, which is consistent with Hye Won Lee's team's research of lung cancer that is associated with EMT mechanism of tumor cells [26]. However, according to expression profile, generally a-sma only expressed itself in fibroblasts, smooth muscles and perivascular cells. Therefore, it is necessary for exploring how a-sma regulated the related molecular involving with EMT in the further study.

Undeniable, there are several limitations of this study. Firstly, the sample size of this study is not large enough. Our conclusion needs to be verified in future by a multi-institution investigation. Secondly, retrospective data requires validation by prospective multi-institution study. However, our data revealed that high density of CAFs was significantly associated with clinical outcomes of patients with NPCs, and thus indicated high density of CAFs as a high risk factor for the development of the patients with NPCs.

\section{Acknowledgements}

This study was supported by grant from the Nature Science Foundation of China (No.81672407).

\section{Authors' contributions}

XKZ and XD are responsible for the study design. JWC and PFY performed the experiments and draft the manuscript. YBX, JL, MYC and YJZ participated in the data analysis and interpretation. All authors read and approved the final manuscript.

\section{Competing Interests}

The authors have declared that no competing interest exists.

\section{References}

1. Chang ET and Adami HO. The enigmatic epidemiology of nasopharyngeal carcinoma. Cancer epidemiology, biomarkers \& prevention : a publication of the American Association for Cancer Research, cosponsored by the American Society of Preventive Oncology. 2006; 15(10):1765-1777.

2. Al-Sarraf M and Reddy MS. Nasopharyngeal carcinoma. Current treatment options in oncology. 2002; 3(1):21-32.

3. Fuyuhiro Y, Yashiro M, Noda S, Kashiwagi S, Matsuoka J, Doi Y, Kato Y, Hasegawa T, Sawada T and Hirakawa K. Upregulation of cancer-associated myofibroblasts by TGF-beta from scirrhous gastric carcinoma cells. British journal of cancer. 2011; 105(7):996-1001.

4. Klobukowska HJ and Munday JS. High Numbers of Stromal Cancer-Associated Fibroblasts Are Associated With a Shorter Survival Time in Cats With Oral Squamous Cell Carcinoma. Veterinary pathology. 2016; 53(6):1124-1130.

5. Huang L, Xu AM, Liu S, Liu W and Li TJ. Cancer-associated fibroblasts in digestive tumors. World journal of gastroenterology. 2014; 20(47):17804-17818.

6. Fuyuhiro Y, Yashiro M, Noda S, Kashiwagi S, Matsuoka J, Doi Y, Kato Y, Muguruma K, Sawada T and Hirakawa K. Myofibroblasts are associated with the progression of scirrhous gastric carcinoma. Experimental and therapeutic medicine. 2010; 1(4):547-551.

7. Yamashita M, Ogawa T, Zhang X, Hanamura N, Kashikura Y, Takamura M, Yoneda $\mathrm{M}$ and Shiraishi T. Role of stromal myofibroblasts in invasive breast cancer: stromal expression of alpha-smooth muscle actin correlates with worse clinical outcome. Breast cancer. 2012; 19(2):170-176

8. Wang WQ, Liu L, Xu HX, Luo GP, Chen T, Wu CT, Xu YF, Xu J, Liu C, Zhang $\mathrm{B}$, Long J, Tang ZY and Yu XJ. Intratumoral alpha-SMA enhances the prognostic potency of CD34 associated with maintenance of microvessel integrity in hepatocellular carcinoma and pancreatic cancer. PloS one. 2013; 8(8):e71189.

9. Sekihara K, Hishida T, Ikemura S, Saruwatari K, Morise M, Kuwata T, Fujii S, Kojima M, Ochiai A, Funai K, Aokage K, Yoshida J, Tsuboi M and Ishii G. The association of intravascular stromal cells with prognosis in high-grade neuroendocrine carcinoma of the lung. Journal of cancer research and clinical oncology. 2016; 142(5):905-912.

10. Wang S, Ma N, Kawanishi S, Hiraku Y, Oikawa S, Xie Y, Zhang Z, Huang G and Murata M. Relationships of alpha-SMA-positive fibroblasts and SDF-1-positive tumor cells with neoangiogenesis in nasopharyngeal carcinoma. BioMed research international. 2014; 2014:507353.

11. Lao XM, Liang YJ, Su YX, Zhang SE, Zhou XI and Liao GQ. Distribution and significance of interstitial fibrosis and stroma-infiltrating $\mathrm{B}$ cells in tongue squamous cell carcinoma. Oncology letters. 2016; 11(3):2027-2034.

12. Ding L, Zhang $Z$, Shang $D$, Cheng J, Yuan $H, W u ~ Y$, Song $X$ and Jiang $H$. alpha-Smooth muscle actin-positive myofibroblasts, in association with epithelial-mesenchymal transition and lymphogenesis, is a critical prognostic parameter in patients with oral tongue squamous cell carcinoma. Journal of oral pathology \& medicine : official publication of the International Association of Oral Pathologists and the American Academy of Oral Pathology. 2014; 43(5):335-343. 
13. Thiery JP. Epithelial-mesenchymal transitions in tumour progression. Nature reviews Cancer. 2002; 2(6):442-454.

14. Inoue $H$, Tsuchiya $H$, Miyazaki $Y$, Kikuchi $K$, Ide F, Sakashita $H$ and Kusama K. Podoplanin expressing cancer-associated fibroblasts in oral cancer. Tumour biology : the journal of the International Society for Oncodevelopmental Biology and Medicine. 2014; 35(11):11345-11352.

15. Kalluri R. Basement membranes: structure, assembly and role in tumour angiogenesis. Nature reviews Cancer. 2003; 3(6):422-433.

16. Galie M, Sorrentino C, Montani M, Micossi L, Di Carlo E, D'Antuono T, Calderan L, Marzola P, Benati D, Merigo F, Orlando F, Smorlesi A, Marchini C, Amici A and Sbarbati A. Mammary carcinoma provides highly tumourigenic and invasive reactive stromal cells. Carcinogenesis. 2005; 26(11):1868-1878.

17. Kunz-Schughart LA and Knuechel R. Tumor-associated fibroblasts (part I): Active stromal participants in tumor development and progression? Histology and histopathology. 2002; 17(2):599-621.

18. Cirri $P$ and Chiarugi P. Cancer-associated-fibroblasts and tumour cells: a diabolic liaison driving cancer progression. Cancer metastasis reviews. 2012; 31(1-2):195-208

19. Mantoni TS, Lunardi S, Al-Assar O, Masamune A and Brunner TB. Pancreatic stellate cells radioprotect pancreatic cancer cells through beta1-integrin signaling. Cancer research. 2011; 71(10):3453-3458.

20. Cheng Y, Wang K, Ma W, Zhang X, Song Y, Wang J, Wang N, Song Q, Cao F, Tan B and Yu J. Cancer-associated fibroblasts are associated with poor prognosis in esophageal squamous cell carcinoma after surgery. International journal of clinical and experimental medicine. 2015; 8(2):1896-1903.

21. Saigusa S, Toiyama Y, Tanaka K, Yokoe T, Okugawa Y, Fujikawa H, Matsusita K, Kawamura M, Inoue Y, Miki C and Kusunoki M. Cancer-associated fibroblasts correlate with poor prognosis in rectal cancer after chemoradiotherapy. International journal of oncology. 2011; 38(3):655-663.

22. Bello IO, Vered M, Dayan D, Dobriyan A, Yahalom R, Alanen K, Nieminen P, Kantola S, Laara E and Salo T. Cancer-associated fibroblasts, a parameter of the tumor microenvironment, overcomes carcinoma-associated parameters in the prognosis of patients with mobile tongue cancer. Oral oncology. 2011; 47(1):33-38.

23. Tsujino T, Seshimo I, Yamamoto H, Ngan CY, Ezumi K, Takemasa I, Ikeda M, Sekimoto M, Matsuura N and Monden M. Stromal myofibroblasts predict disease recurrence for colorectal cancer. Clinical cancer research : an official journal of the American Association for Cancer Research. 2007; 13(7):2082-2090.

24. Surowiak P, Murawa D, Materna V, Maciejczyk A, Pudelko M, Ciesla S, Breborowicz J, Murawa P, Zabel M, Dietel M and Lage H. Occurence of stromal myofibroblasts in the invasive ductal breast cancer tissue is an unfavourable prognostic factor. Anticancer research. 2007; 27(4C):2917-2924.

25. Mertens JC, Fingas CD, Christensen JD, Smoot RL, Bronk SF, Werneburg NW, Gustafson MP, Dietz AB, Roberts LR, Sirica AE and Gores GJ. Therapeutic effects of deleting cancer-associated fibroblasts in cholangiocarcinoma. Cancer research. 2013; 73(2):897-907.

26. Lee HW, Park YM, Lee SJ, Cho HJ, Kim DH, Lee JI, Kang MS, Seol HJ, Shim YM, Nam DH, Kim HH and Joo KM. Alpha-smooth muscle actin (ACTA2) is required for metastatic potential of human lung adenocarcinoma. Clinical cancer research : an official journal of the American Association for Cancer Research. 2013; 19(21):5879-5889. 